\title{
Radiofrequency Lesioning of the L2 Ramus Communicans in Managing Discogenic Low Back Pain
}

Thomas T. Simopoulos, MD, Atif B. Malik, MD, Khuram A. Sial, MD, Mohamed Elkersh, MD, and

Zahid H. Bajwa, MD

Background : Discogenic low back pain is a common cause of chronic low back pain that remains a treatment challenge. The innervation and transmission of nociceptive information from painful lumbar discs has only recently been better described.

Objective: To report initial experience of effectiveness of radiofrequency lesioning of $L 2$ ramus communicans in managing discogenic pain.

Study Design : A prospective, case series.

Methods: A case series of 5 patients who had radiofrequency lesioning of the ramus communicans at the L2 level. All pa- tients had discogenic low back pain and had diagnostic blocks with local anesthetic at the level of the $L 2$ ramus communicans demonstrating significant pain relief. Continuous radiofrequency lesioning at $80^{\circ} \mathrm{C}$ of the L2 ramus communicans for 60 seconds was performed.

Standard outcome measures of reduction in the visual analogue scale (VAS), improvement in function, reduction in pain medication, and consistent improvement in low back pain with repeating of the procedure after its initial effect has worn off were recorded.

Results : All five patients had consis- tent pain relief after a minimum of 2 radiofrequency lesioning treatments approximately 4 months apart. Four of the five patients had a reduction in pain medication, and all reported improvement in sitting tolerance and functioning. There were no side effects or complications.

Conclusion: Radiofrequency lesioning of the $L 2$ ramus communicans seems to offer partial relief for patient's suffering from discogenic pain. Further studies are needed to confirm our results.

Keywords: discogenic low back pain, radiofrequency lesioning, ramus communicans, discogenic low back pain
Chronic discogenic low back pain is a major problem in many industrialized nations. Internal disc disruption (IDD) is thought to account for $40 \%$ of cases of chronic low back pain (1). The treatment of this entity remains contentious, and no one modality has had prevailing success. Even more controversial is the treatment of IDD in the presence of previous lumbar spine surgery or coexisting pathology such as facet disease. To complicate matters further, third party payers often do not reimburse newer procedures designed to directly address IDD, citing inadequate data.

The mechanism of pain from IDD and its transmission is not completely elucidated. It is well accepted that the outer one-third of the annulus fibrosis receives rich sensory innervation. Abnor-

From Beth Israel Deaconess Hospital and Harvard Medical School Boston, MA.

Address Correspondence: Atif Malik, MD, 330

Brookline Ave. Boston, MA 02215

Disclaimer: None of the authors have any disclosures or grants.

Conflict of Interest: None

Acknowledgement:

Manuscript received on 8/9/2004

Revision submitted on 12/13/2004

Accepted for publication on $12 / 15 / 2004$ mal nerve in-growth and sensitization of nociceptors in the annulus fibrosis have been thought to distinguish a painful IDD from a painless one (2). How this nociceptive information is transmitted into the central nervous system in humans is not clear. In the rat, sensory information from the lumbar intervertebral discs is transmitted from the sinuvertebral nerve into the rami communicans on each side of a given disc. The rami communicans then preferably connect to the paravertebral sympathetic chain (3). This afferent information then is transferred via the $\mathrm{L} 1$ and L2 communicating rami to the dorsal root ganglia of L1 and L2, and subsequently to the spinal cord.

In humans, the sinuvertebral nerve is thought to be a mixed nerve formed by a somatic root from the ventral ramus as well as from the ramus communicans (4). However, according to Nakamura et al (5), the origin of the nerves innervating the posterior aspect of the intervertebral disc is the sympathetic trunk. They postulated that the neuroanatomy for the transmission of the pain for IDD is similar to the pathways described in rats. They obtained good results from the injection of $1 \% \mathrm{li}$ docaine into the $\mathrm{L} 2$ nerve root in patients with L4/5 and L5/S1 discogenic pain.

In our clinic, we had obtained good but transient relief of chronic discogenic low back pain with local anesthetic blockade of either the L2 nerve root or the L2 communicating ramus. We report our initial experience in interrupting the transmission of nociceptive impulses along the L2 rami communicans using continuous radiofrequency lesioning (RFL) in an effort to provide longterm management of refractory discogenic low back pain.

\section{Methods}

In our cases series we chose patients who complained of intense back pain in the belt line distribution. Some cases involved radiation to the hips and/or buttocks. In all cases, patients suffered from significant sitting intolerance (15 to 30 minutes maximum). Pain patterns were similar to those described by Nakamura et al (5). All other sources of low back pain including facet disease, sacroiliac joint pain, spinal instability, and significant myofascial pain were either treated successfully or excluded from this series. Patients with psychological problems were also carefully screened out. 
The presumed clinical diagnosis of discogenic low back pain was made by history, physical examination, and Magnetic Resonance Imaging (MRI). Our patients had significant pain provocation with forward flexion, and sitting intolerance. Prior to treatment, the physical examination was unremarkable for signs of nerve root involvement or other sources of low back pain. Patients that had extensive spondylosis, spondylolisthesis, instrumentation, or stenosis were not candidates for L2 ramus communicans lesioning. Provocative discography with concordant pain on injection confirmed the diagnosis of discogenic pain. In addition, the correlation of discogenic pain to the L2 ramus communicans was established via a diagnostic local anesthetic block, prior to RF lesioning. For unilateral low back pain, the corresponding L2 ramus communicans was blocked and for bilateral pain, both sides were anesthetized with $0.5 \mathrm{ml}$ of $0.5 \%$ bupivacaine. All of the patients in this series reported greater than $50 \%$ alleviation of their back pain with much improved sitting tolerance. They were then selected as candidates for RF lesioning.

Patients were NPO on the day of the procedure. After intravenous access was obtained and standard ASA monitors placed, a light sedative, midazolam was given. On the fluoroscopy table, patients were placed in the prone position with a

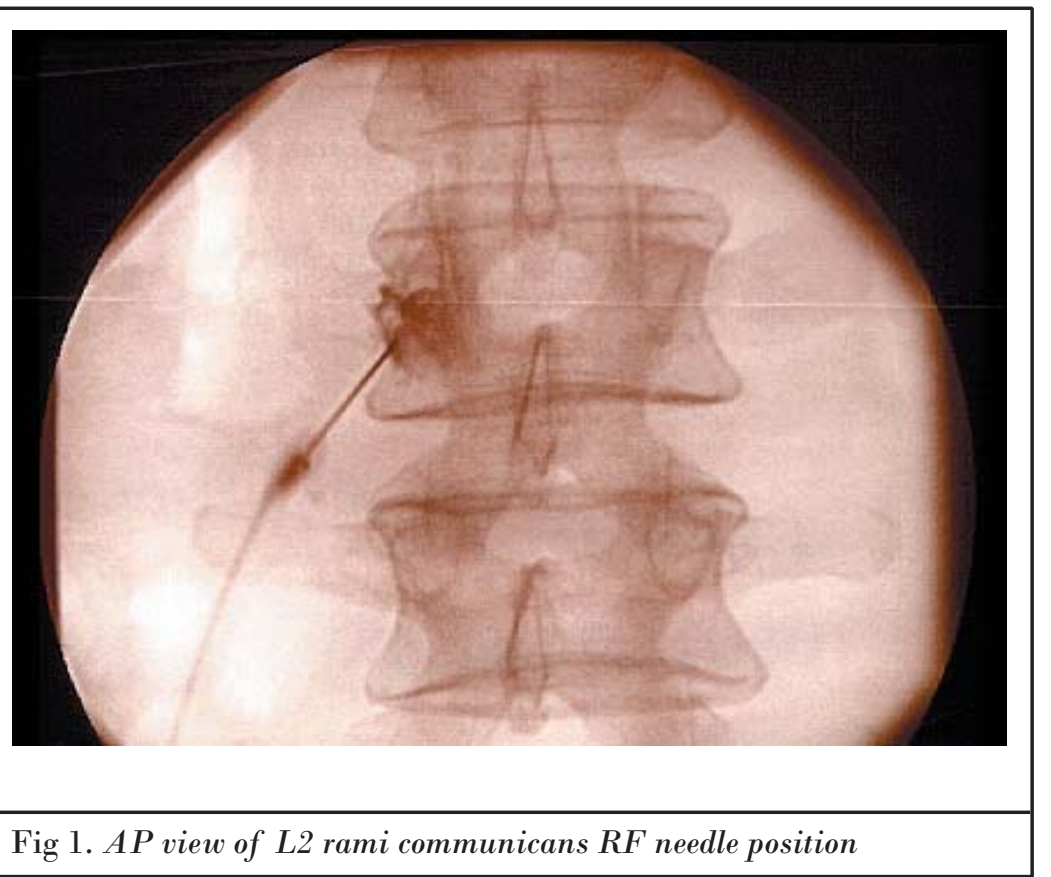

pillow under the abdomen. $\mathrm{C}$ arm fluoroscopy was used to locate the L2 vertebral body and the endplates were squared. Two views (an AP view and oblique view) were then used to place at least $10 \mathrm{~mm}$ of the tip of a curved 5 inch 22 gauge spinal needle against the vertebral body. The oblique view involved $\mathrm{C}$ arm rotation to approximately 30 degrees toward the side to be anesthetized. The puncture site is typically $6-7 \mathrm{~cm}$ from midline. The needle is advanced to the middle to caudal one third of the height of the body. The tip of the needle is not past the middle of the length of the body so as to avoid the sympathetic chain. The needle should contact periosteum. Contrast material is then injected to ensure the needle is not in psoas muscle or intravascular.

After a thorough explanation to the patients, consent was obtained for percutaneous radiofrequency of the L2 ramus communicans. The technique used for positioning of the RF cannula is identical to the diagnostic nerve block. After a 20 gauge, $15 \mathrm{~cm}$ length, $10 \mathrm{~mm}$ active tip, bent SMK-C 15 needle (Radionics, Tyco Health Care Group, 22 Terry Avenue, Burlington, MA, 01803) was placed in the proper position, the stylet was replaced by the RF probe, and was connected to the RFG-3B generator (Radionics, Burlington MA). Final needle position was verified with AP and lateral views (Figs. 1 and
2). Sensory stimulation at $50 \mathrm{~Hz}, 0.2$ millisecond pulse width, was performed and provoked concordant deep aching pain in the low back with variable reproduction in the hip or buttock area at $0.5 \mathrm{~V}+/$ $0.1 \mathrm{~V}$. Stimulation of the neighboring genitofemoral nerve was excluded by contact with vertebral body and changing needle position if paresthesias occurred in the groin. Contrast medium was also used to confirm needle position was not in striated psoas muscle. Contrast also aided in exclusion of accidental cannulation of a vertebral body vein. The RF cannula was determined to be safely away from motor nerves after stimulation at a frequency of $2 \mathrm{~Hz}$ and the voltage set to 3 . RF lesions were done at $80^{\circ} \mathrm{C}$ for 60 seconds after the injection of $1 \mathrm{cc}$ of $2 \%$ lidocaine with 10 mg methylprednisolone acetate. The purpose of the deposteroid was to prevent post-procedure neuritis. RF lesioning was performed on both sides at the L2 level for bilateral discogenic low back pain, and unilaterally if the pain affected primarily one side.

The outcome measures determined in this case series were: (1) The visual analog scale (VAS); this is a single question comprised of a 100-mm line anchored with "no pain" and "the worst pain possible." To quantify the rating from 0 to 10 , the respondent places a mark across the line at the point that represents perceived pain intensity. A reduction of the preprocedure pain score by at least $50 \%$ was defined as effective. The duration of relief was prospectively followed. (2) An improvement in function; This information was collected from the patient through a series of questions where the patient reported improved sitting/standing tolerance. There was also an evaluation on the activities of daily living. (3) Decrease on the use of pain medication was also used as an indicator of overall improvement. (4) Finally, the willingness to repeat the procedure, and the avoidance of other procedures or surgery was used to evaluate the overall long-term effectiveness.

\section{CASE Reports}

\section{Case 1}

A 37 year-old female otherwise healthy with a 10-year history of low back pain had been referred to our center status post L4/ 5 and L5/S1 discectomy in 1994. She was treated in our center with epidural blocks, facet/medial branch blocks, and later 


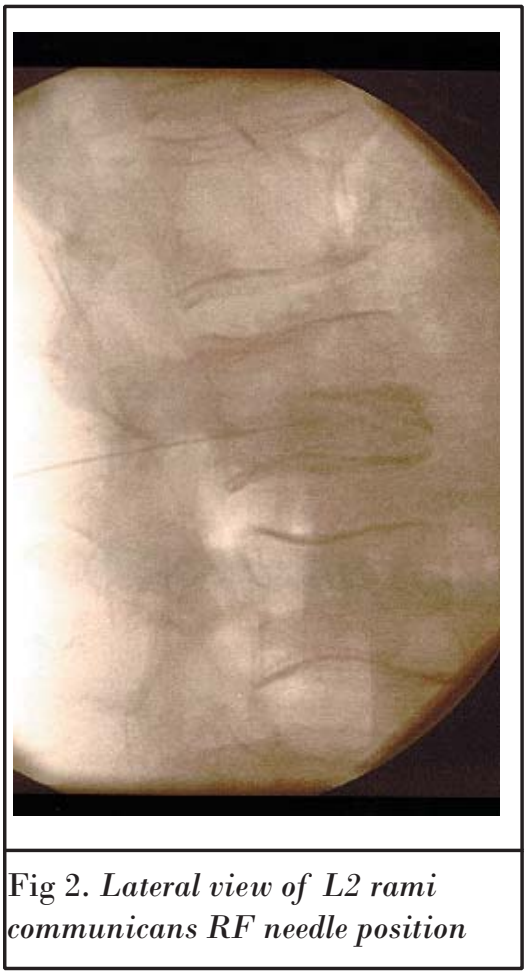

radiofrequency lesioning of the lumbar medial branches. She had some modest improvement but continued to require sustained release oxycodone $20 \mathrm{mg}$ BID and hydrocodone/acetaminophen 5/325 3 times a day. She complained of left-sided belt-line pain with minimal radiation to the buttock area and sitting intolerance. Her physical exam revealed mild loss of lumbar lordosis, mild paraspinous muscle tenderness, and a well healed surgical scar. The neurological examination was non-focal. MRI scanning disclosed minimal bulging at L4/L5 and a previous laminectomy. Concordant pain at this level was demonstrated by provocative discography. An L2 ramus communicans block gave her excellent temporary improvement (VAS reduction by $85 \%)$. On stimulation of the ramus communicans, she had reproduction of her low back pain. She subsequently had radiofrequency denervation of this region with 3 months of pain relief. Her sitting intolerance increased from 15 minutes to 43 minutes. Her VAS score was reduced from $7 / 10$ to $3 / 10$. The patient stopped her OxyContin and remained on 2 hydrocodone/ acetaminophen tablets per day. She presented 4 months post-radiofrequency for a repeat $\mathrm{L} 2$ communicans denervation with consistently good relief. A third radiofrequency procedure 4 months later was postponed because she became pregnant.

\section{Case 2}

A 45 year old male, exterior house painter with a progressive 5 year history of right sided low back radiating to the groin reported no sustained benefit after a series of 3 lumbar epidural steroid injections. His past medical history was significant for hepatitis $\mathrm{C}$, reflux disease and migraine. His physical exam was essentially positive only for paraspinous muscle tenderness. He had a negative scrotal ultrasound. His MRI scan revealed a small disc protrusion to the right with no nerve root impingement. Replication of the patient's chronic pain was obtained after discography at the L4/5 level. After a diagnostic block at L2 ramus communicans rendered the patient with greater than $50 \%$ relief, radiofrequency lesioning was performed during a subsequent visit. He had a reduction in his VAS from 8/10 to $4 / 10$. He went off his sustained oxycodone medication. A repeat radiofrequency lesion was done approximately 5 months later. He has returned to prior work, and uses analgesics as needed.

\section{Case 3}

A 38 year-old HIV positive male presented to our center 2 years after an L5/ S1 laminectomy failed to control rightsided radicular pain. He had multiple nerve blocks, epidural trials, and medications with only modest benefit. A spinal cord stimulator was placed with excellent control of right leg pain. However he had persistent low back pain that was significantly worse with sitting. His physical examination was significant for hypesthesia in the right S1 dermatome, reduced ankle reflex on the right, and paraspinous muscle tenderness of the right, greater than left side. Facet and sacroiliac joint injections as well as trigger point injections were of minimal benefit. On provocative discography, his pain was reproduced on injection of the L5/S1 disc. RF of the L2 ramus communicans after successful local anesthetic block afforded him with good relief for 4 months. His VAS scores were reduced from $6 / 10$ to $2 / 10$. He presented again for the treatment and has had sustained benefit to this date. He has been able to retain his job as an employee in a grocery store and play baseball. He was not able to reduce his methadone at 10 mg, 3 times a day.

\section{Case 4}

A 57 year-old female presented to our center with a one-year history of right-sided low back pain radiating to the buttock, hip, and groin and right radicular leg pain. Her past medical history was significant for reflux disease and migraines. She had mild hypoesthesia in a L4 distribution. Her knee and ankle reflexes were symmetrical, and her motor exam was without weakness. Her gait was antalgic, no Romberg, and vibratory sense was decreased in the toes. Her MRI was positive for a mild right-sided L3/L4 disc herniation without direct nerve root involvement. A L4 nerve root block resolved her radicular pain but she still suffered from groin pain and sitting intolerance. Provocation of the L3/4 disc was concordant with her pain. A diagnostic ramus communicans block at the L2 level reduced her pain from 6/10 to a VAS of 1/10. Stimulation of the L2 communicans duplicated her pain in the low back with radiation to the buttock and hip. She had $50 \%$ reduction in her VAS for the next 3 months. She then received a repeat procedure and has had persistent relief for 4 months. She has been able to manage her pain with acupuncture and as needed, rocefecoxib. Her regular use of tramadol was drastically reduced to only episodic use. She reported fewer days where she needed to leave work early.

\section{Case 5}

A 57 year old male with a past medical history hypercholesterolemia, hypertension, peptic ulcer disease, and migraines presented to our center complaining of an 8 year history of progressive low back pain at the waist line region. He stated that bending forward provoked his pain as well as extension. He had tried chiropractic therapy without significant improvement. His medication included celecoxib $100 \mathrm{mg}$ BID, gabapentin $300 \mathrm{mg}$ qhs, and propoxyphene/acetaminophen $5 \mathrm{mg} / 325 \mathrm{mg}$ (2-4 tablets/day). An MRI scan demonstrated grade I anterolisthesis at L4/L5, an L4/L5 disc bulge, modest disc space narrowing at L4/L5, and facet hypertrophy at L4/L5. Previous epidural injections, facet blocks, and sacroiliac joint blocks rendered no benefit. Even during 
anesthetic phase of the latter 2 types of injections, no improvement was found. The patient began to consider surgical fusion.

He then had provocative discography, which was concordant at the L4/L5 level. A diagnostic bilateral L2 ramus communicans provided $70 \%$ relief of his low back pain symptoms. He has been treated with radiofrequency neurolysis of the $\mathrm{L} 2$ rami communicans for one and a half years. He has been able to avoid fusion. Yearly plain films have demonstrated a stable grade I anterolisthesis. His relief lasts between 3-4 months. The reduction in his VAS scores has ranged from 50 to $80 \%$. He reports being able to do more physical labor after RFL. His analgesic consumption decreased to one half the usual quantity after treatment.

\section{RESULTS}

All five patients had consistent pain relief after a minimum of 2 radiofrequency lesioning treatments approximately 4 months apart. Four of the five patients had a reduction in pain medication, and all reported improvement in sitting tolerance and functioning. There were no side effects or complications.

\section{DisCUSSION}

The intervertebral disc has long been regarded as the main source of pain in chronic low back pain. The medical treatment options for patients with chronic discogenic low back pain have included physical therapy, lumbar corsets, manual manipulation, back school, life style modification and oral medications. However, many patients continue to have sub-optimal results with persistent pain. In addition, it is not uncommon for patients to have epidural or intradiscal steroid injections with only transient benefit Indeed, the prognosis for chronic discogenic low back pain is not reassuring (6).

Surgical fusion has long been regarded as the "gold standard" of treatment for discogenic pain. But, in a recent review by Deyo et al (7), the most controversial use of spinal fusion is for the treatment of chronic discogenic low back pain. The morbidity and failure rates of spinal fusion have prompted the emergence of minimally invasive therapeutic options. Intradiscal electrothermal therapy (IDET) is an intradiscal annular heating method, which has been shown to generate temperatures sufficient to lesion nerve fibers and denature collagen (8). Collagen remodeling is thought to stabilize annular fissures with less intradiscal pressure. Applying IDET to the pathological entity IDD makes good theoretical sense, and offers an alternative to intervertebral fusion.

Unfortunately, follow-up studies after treatment with IDET have yielded varying outcomes: $16-63 \%$ success rate. Investigators often site the need for improvement of patient selection among those with chronic discogenic low back pain (9-11). Other authors argue that the IDET procedure cannot generate temperatures high enough to alter collagen architecture (12-13). Despite a pathophysiologic description of IDD, and a modality geared towards discogenic pain, the treatment of this entity is far from curative.

More recently, a randomized control trial by Oh and Shim (14) evaluated the effectiveness of radiofrequency denervation of the ramus communicans for chronic pain related to IDD. This small study selected patients with chronic discogenic low back pain of the L3/4 or L4/ 5 level who have significant pain persisting after IDET. Patients randomized to the treatment group received thermocoagulation of the ramus communicans above and below the vertebrae of the affected disc on the painful side or bilaterally if pain complaints involved both sides. The control group received a similar needle placement but only an injection of lidocaine without radiofrequency neurotomy. At 4-month follow-up, patient with radiofrequency denervation of the ramus communicans reported favorable results compared to the control group. The authors concluded that this may be a safe option for the palliation of chronic intractable disc related low back pain. They did not treat L5/S1 disc related pain, which is a commonly affected disc.

Despite extensive investigation on the pathology of IDD, the anatomical transmission of nociceptive information from the vertebral disc is incompletely understood. Presently, an increasingly preferential hypothesis is that the sinuvertebral nerves are branches of the rami communicans, which ultimately relay noxious information into the lumbar sympathetic trunk. Although the sinuvertebral nerve is frequently thought to receive contributions from the ventral ramus of the corresponding nerve root, Groen et al (15) have shown that the sinuvertebral nerve originates only from the ramus communicans in human. This neuroanatomic relationship has been shown to be the case in rats as well (3). Nakamura et al (5) have postulated that the sympathetic trunk is the origin of the innervation of the posterior aspect of the intervertebral disc. They suggested that the pain of IDD is transmitted non-segmentally by visceral sympathetic afferents mainly to the L2 (variable contribution of L1) root via the communicans nerves at this level. They further postulated that infiltration of the L2 root with local anesthetic maybe used as a conservative diagnostic tool for discogenic pain. The distance of the L2 root or communicating ramus from pain generators in the waistline area may offer the advantage of not anesthetizing more than one source of pain. For example, the blockade of medial branches to lumbar facets in the L4-S1 region in clinical practice can easily result in the anesthetization of painful muscles or ligaments in the neighboring region.

Chronic discogenic low back pain remains a common and challenging pathological entity to treat. IDD may be a cause for persistent pain after laminectomy or fusion. IDD may also coexist with other causes of low back pain such as facet disease or spondylolisthesis. Our group of patients represents a small spectrum of the coexisting complexities that often accompanies patients with chronic IDD. Prior back surgery, facet disease, severe radiculopathy, and spondylolisthesis often accompany IDD. The partial relief obtained in this challenging group of patient suggests that the ramus communicans at the L2 level may play a role in pain transmission from IDD at lower levels. The fact that pain relief was not complete may represent partial denervation of the communicating nerves or the L1 nerve root may also receive nociceptive information from the sympathetic chain. Further work is necessary to understand the transmission of pain and the application of RFL to such pathways.

\section{Conclusion}

Radiofrequency lesioning of the L2 ramus communicans can improve pain control in patients with severe discogenic pain. Fluoroscopy and electrical stimulation are required to safely and effectively carry out the procedure. This technique is easy to repeat and appears to be effective over time. 


\section{Author Affiliation}

Thomas T. Simopoulos, MD

Clinical Instructor

Department of Anesthesia

Beth Israel Deaconess Hospital

Harvard Medical School

330 Brookline Ave

Boston, MA 02215

tsimopolous@bidmc.harvard.edu

Atif B. Malik, MD

Department of Anesthesia

Beth Israel Deaconess Hospital

Harvard Medical School

330 Brookline Ave

Boston, MA 02215

E-mail: amalik@bidmc.harvard.edu

Khuram A. Sial, MD

Pain Fellow

Department of Anesthesia

Beth Israel Deaconess Hospital

Harvard Medical School

330 Brookline Ave

Boston, MA 02215

E-mail: ksial@bidmc.harvard.edu

\section{Mohamed Elkersh, MD}

Department of Anesthesia

Beth Israel Deaconess Hospital

Harvard Medical School

330 Brookline Ave

Boston, MA 02215

E-mail: elkershmd@hotmail.com

\section{Zahid H. Bajwa, MD}

Assistant Professor

Department of Anesthesia \&

Neurology

Beth Israel Deaconess Hospital

Harvard Medical School

330 Brookline Ave

Boston, MA 02215

E-mail: zbajwa@bidmc.harvard.edu

\section{REFERENCES}

1. Schwarzer AC, Aprill CN, Derby R, Fortin J, Kine G, Bogduk N. The prevalence and clinical features of internal disc disruption in patients with chronic low back pain. Spine 1995; 20:1878-1883.

2. Freemont AJ, Peacock TE, Goupille P, Hoyland JA, O’Brien J, Jayson MI. Nerve ingrowth into disease intervertebral disc in chronic back pain. Lancet 1997; 350:178 181.

3. Kaoru S, Takahashi Y, Takahashi K et al. Sensory nerve fibers from lumbar intervertebral discs pass through rami communicantes: A possible pathway for discogenic low back pain. J Bone Joint Surg (Br) 1998; 8:737-742.

4. Sehgal N, Fortin JD. Internal disc disruption and low back pain. Pain Physician 2000; 3:143-157.

5. Nakamura SI, Takahashi K, Takahashi Y, Yamagata M, Moriya $\mathrm{H}$. The afferent pathways of discogenic low-back pain: Evaluation of L2 spinal nerve infiltration. J Bone Joint Surg (Br) 1996; 78:606-612.

6. Karasek M, Bogduk N. Twelve-month follow-up of a controlled trial of intradiscal thermal anuloplasty for back pain due to internal disc disruption. Spine 2000; 25: 2601-2607.

7. Deyo RA, Nachemson A, Mirza SK. Spinalfusion surgery: The case for restraint. $N$ Eng J Med 2004; 350:722-726.

8. Saal JS, Saal JA. Management of chronic discogenic low back pain with a thermal intradiscal catheter: A preliminary report. Spine 2000; 25:382-388.

9. Lee MS, Cooper G, Lutz GE, Lutz C, Hong HM. Intradiscal electrothermal therapy (IDET) for treatment of chronic lumbar discogenic pain: A minimum of 2-year clinical outcome study. Pain Physician 2003; 6:443-448.

10. Derby R, Eck B, Chen Y, O'Neill C, Ryan D. Intradiscal electrothermal annuloplasty (IDET): A novel approach for treating chronic discogenic back pain. Neuromodulation 2000; 3:69-75.

12. Pauza KJ, Howell S, Dreyfuss P. A randomized, placebo-controlled trial of intradiscal electrothermal therapy for the treatment of discogenic low back pain. Spine J 2004;
4:27-35.

13. Kleinstueck FS, Diederich CJ, Nau WH, Puttlitz CM, Smith JA, Bradford DS, Lotz JC. Acute biochemical and histological effects of intradiscal electrothermal therapy on human lumbar discs. Spine 2001; 26: 2198-2207.

14. Oh WS, Shim JC. A randomized controlled trial of radiofrequency denervation of the ramus communicans nerve for chronic discogenic low back pain. Clin J Pain 2004; 20:55-60.

15. Groen GJ, Baljet B, Drukker J. Nerves and nerve plexuses of the human vertebral column. Am J Anat 1990; 188:286-296. 
